\title{
A Web based Multi-Linguists Symbol-to-Text AAC Application
}

\author{
Chaohai Ding \\ Web and Internet Science Group \\ University of Southampton \\ Southampton, SO17 1BJ, UK \\ cd8e10@ecs.soton.ac.uk \\ Yunjia Li \\ Web and Internet Science Group \\ University of Southampton \\ Southampton, SO17 1BJ, UK \\ yl2@ecs.soton.ac.uk
}

\author{
Nawar Halabi \\ Web and Internet Science Group \\ University of Southampton \\ Southampton, SO17 1BJ, UK \\ nawar.halabi@gmail.com
}

E.A. Draffan
Web and Internet Science Group
University of Southampton
Southampton, SO17 1BJ, UK
ead@ecs.soton.ac.uk

\author{
Lama Al-Zaben \\ Web and Internet Science Group \\ University of Southampton \\ Southampton, SO17 1BJ, UK \\ laa1c13@ecs.soton.ac.uk
}

Mike Wald
Web and Internet Science Group
University of Southampton
Southampton, SO17 1BJ, UK
mw@ecs.soton.ac.uk

\begin{abstract}
There are several commercial or freely available symbol sets for Augmentative and Alternative Communication (AAC) use; all these symbol sets have the same issue when trying to use them in a multiple lingual setting. Symbol Dragoman is a Web based application that aims to allow the user who has no spoken language and uses pictograms or images to communicate in Arabic or English. It combines chosen 'symbols' in any way they want to produce a sentence that can be read or heard in both languages with the potential of offering any combination of languages in the future.
\end{abstract}

\section{Categories and Subject Descriptors}

K.4.2 [Computer and Society]: Social Issues - Assistive technologies for persons with disabilities; H.3.5 [On-line Information Services]: Web-based services; H.5.2 [Information Interfaces and Presentation]: User Interfaces-Input devices and strategies.

\section{General Terms}

Design, Human Factors

\section{Keywords}

AAC, Accessibility, Symbol-to-Text Communication

\section{INTRODUCTION}

There are several commercial or freely available symbol sets for Augmentative and Alternative Communication (AAC) use; all have the same issue when trying to use them in a multi lingual setting. Each symbol is either tied to a particular word or a multiword phrase e.g. 'put' versus 'put on', 'put off', 'put through' and 'put down'. Often the multiword phrase encourages the symbol designer to include an image of an object such as 'a box' - so the image could represent 'put on the box' or just 'put on' or even just 'put'. The problem is that we need a system that not only translates the symbol into a word or multiword in both Arabic and English but also allows the user to build a sentence in

Permission to make digital or hard copies of part or all of this work for personal or classroom use is granted without fee provided that copies are not made or distributed for profit or commercial advantage and that copies bear this notice and the full citation on the first page. Copyrights for third-party components of this work must be honored. For all other uses, contact the Owner/Author.

Copyright is held by the owner/author(s). $W 4 A$ '15, May 18-20, 2015, Florence, Italy ACM 978-1-4503-3342-9/15/05.

http://dx.doi.org/10.1145/2745555.2746674 symbols that can be recognised in both languages.

\section{CHALLENGES AND SOLUTIONS}

One of the main challenges for translating from symbols to text is that the input may lack function words, as a user may not use symbols that correspond to function words. A predictive system has been used in a symbol-to-English translation system [7], which assumed that the input is an ordered set of symbols that follows English syntax. The goal of this system is to translate a sequence of symbols to a full English sentence by using a predictive system to anticipate function words that a user may miss.

Another challenge for translation is the order of input symbols. A non-literate individual may not be able to follow the natural language syntax when constructing a message. One solution is simplifying the message construction by using a non-linear construction suggested by Patel et al. [5]. The non-linear construction area is based on a semantic relationship by arranging symbols in a two-dimensional space with a specific location for each semantic role. There are some additional features added to guide the user's input, such as colour code, symbol highlighting or darkening for different purposes.

Another solution is using semantic frames to predict the order presented in SymbolPath [8], [9]. SymbolPath does not provide guidance while constructing a symbol message. Instead, it focuses on minimising the user's effort when constructing a message. Due to the input method - a path that passes by selected symbols - it is assumed that additional unintended symbols are selected. To determine the intended set they used semantic-grams, which are similar to n-grams but not directional and words within each set may not be adjacent, but do need to belong to the same sentence. The position of the path against an icon is also considered.

\section{OVERVIEW OF SYMBOL DRAGOMAN}

Symbol Dragoman (shown in Figure 1), is a Web based application that aims to allow someone who has no spoken language and uses pictograms or images to communicate in Arabic or English. It combines chosen 'symbols' in any way they want to produce a sentence that can be read or heard in both languages. In our system, when converting a relatively short sequence of symbols to one or more meaningful and grammatically well-formed sentences, the AAC user gets to choose one of them to convey the message intended. This helps the user learn more about the vocabulary and syntax of the 
language converted to as they are receiving a mapping from symbols which they know the meaning of to a textual transcript that contains words that describe those symbols. There are two different approaches for generating English and Arabic sentence generation illustrated in Figure 1.

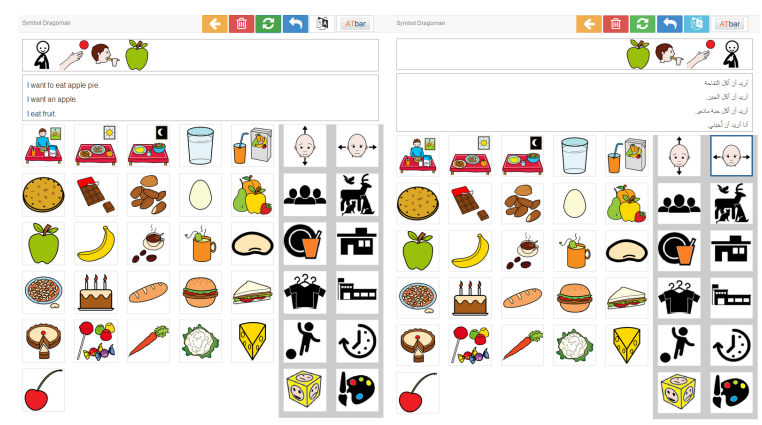

(a)

(b)

Figure 1: User Interface of Symbol Dragoman in both English (a) and Arabic (b)

English Sentence Generation. For English we simply used a large corpus [3] that contains millions of various length sentences only a portion of which are translated to Arabic. The sentences were indexed using elastic search. Then for each symbol, we attached one or more keywords extracted from the ARASAAC symbol dictionary [1]. Optionally, a morphological analysis could be conducted on each word to generate different derivations of the same word.

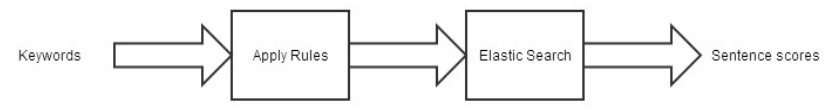

Figure 2: Search Process for English Sentence Generation

The search process for English simply involves using the keywords for each of the requested symbols to search through the indexed sentence corpus as presented in Figure 2. The order of the symbols does not matter here. This generated satisfactory results for English as the corpus is significant but did not work in Arabic as the sentence corpus available from the same source is relatively small ( $\sim 9000$ sentences paralleled with English sentence) and we have no knowledge of any other corpus available with the same characteristics. In order to fix this issue we adopted a different approach for Arabic.

Arabic Sentence Generation. Since Arabic is a highly morphological and derivational language, and pronouns are most of the time realised as word affixes [2], it is difficult to map each symbol to one keyword. For example, the word " "قحافت" which means "an apple" becomes "ي "w when affixed with the first-person possessive pronoun and become "my apple". To fix this we have done the following manually for each symbol's keyword:

1. For verbs: we generated conjugations (feminine and masculine) with all possible subject pronouns attached for (first, second, feminine, masculine, singular and plural). These could be generated automatically but were done manually for our symbol set.

2. For nouns: We left them as they are for the purpose of this demo. Affixing nouns is syntactically and semantically complex. We think it could also be done automatically using a data set like the Buckwalter dictionary but this is left as future work.

3. For adjectives: Four morphs were used for each Adjective keyword (feminine, masculine, singular and plural).

4. All other keywords were considered particles and we used one keyword for each with some exceptions.

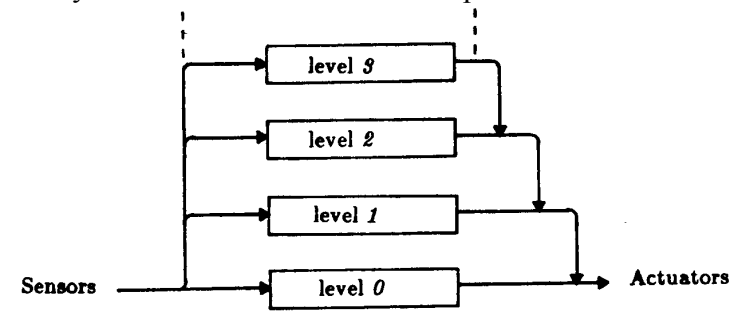

Figure 3: Subsumption Architecture [6]

\section{CONCLUSION}

In summary, in this paper, we present the Symbol Dragoman, a Web based application that aims to allow the user who has no spoken language and uses pictograms or images to communicate in Arabic or English. This application allows Arabic symbol users to work in both languages with the symbols and words appearing from right to left for Arabic and left to right for English. This application is also designed with responsive UI, to fit the screen of mobiles or tablet browsers. As building on the Arabic Symbol Dictionary project, this paper demonstrates the challenges and solutions of AAC when it comes across different languages and cultures. However, there are some limitations for current work, such as the accuracy of the sentence generation, the speed of symbol input and the semantic relations of symbols. In our future work, we would also involve personalization and machine learning to improve the accuracy of sentence generation and the prediction of the symbols input.

\section{REFERENCES}

[1] ARASAAC Symbol Dictionary: http://www.catedu.es/arasaac/index.php.

[2] Habash, N.Y. 2010. Introduction to Arabic Natural Language Processing. Synthesis Lectures on Human Language Technologies. 3, 1 (Jan. 2010), 1-187.

[3] Many Things: http://www.manythings.org/anki/.

[4] Noun Project: http://thenounproject.com/.

[5] R Patel, S Pilato, D.R. 2004. Beyond Linear Syntax: An Image-Oriented Communication Aid. Assistive Technology Outcomes and Benefits (2004).

[6] Subsumption architecture: http://cs.brown.edu/ tld/courses/cs148/02/architectures. html.

[7] Waller, A. et al. 2002. A predictive Blissymbolic to English translation system. Assets. (2002), 186.

[8] Wiegand, K. and Patel, R. 2012. Non-syntactic word prediction for AAC. SLPAT '12 Proceedings of the Third Workshop on Speech and Language Processing for Assistive Technologies. (2012), 28-36.

[9] Wiegand, K. and Patel, R. 2012. SymbolPath: A Continuous Motion Overlay Module for Icon-based Assistive Communication. Proceedings of the 14th International ACM SIGACCESS Conference on Computers and Accessibility. (2012), 209-210. 